\title{
Multiple time scales in volatility and leverage correlations: An stochastic volatility model
}

\author{
Josep Perelló*, Jaume Masoliver ${ }^{\dagger}$, \\ Departament de Física Fonamental, Universitat de Barcelona, \\ Diagonal, 647, E-08028 Barcelona, Spain \\ and Jean-Philippe Bouchaud ${ }^{\ddagger}$ \\ Service the Physique de l'État Condensé, Centre d'Études de Saclay, \\ Orme des Merisiers, 91191 Gif-sur-Yvette Cedex, France and \\ Science and Finance-CFM, 109-111 rue Victor Hugo, 92532 France
}

(Dated: May 3, 2019)

\begin{abstract}
Financial time series exhibit two different type of non linear correlations: (i) volatility autocorrelations that have a very long range memory, on the order of years, and (ii) asymmetric return-volatility (or 'leverage') correlations that are much shorter ranged. Different stochastic volatility models have been proposed in the past to account for both these correlations. However, in these models, the decay of the correlations is exponential, with a single time scale for both the volatility and the leverage correlations, at variance with observations. We extend the linear Ornstein-Uhlenbeck stochastic volatility model by assuming that the mean reverting level is itself random. We find that the resulting three-dimensional diffusion process can account for different correlation time scales. We show that the results are in good agreement with a century of the Dow Jones index daily returns (1900-2000), with the exception of crash days.

PACS numbers: 89.65.Gh, 02.50.Ey, 05.40.Jc, 05.45.Tp
\end{abstract}

\section{INTRODUCTION}

The interest for financial markets models has considerably increased during the last twenty years. The traditional Black-Scholes Gaussian model is now well known to be inadequate; devising faithful and convenient models for the price processes is essential for risk control, derivative pricing and other trading operations. Data analysis has now become a mandatory step in model building, using the wealth of available tick-by-tick data or high-frequency data [1]. There is now enough data to establish, with statistical accuracy, some fundamental properties of market dynamics. Empirical observations provide a list of universal features that seem to hold in most markets, quite independently of the observation period and of the type of asset. These are the so-called stylized facts (see e.g. 2, 3, 4]), which should be reproduced, at least to some extent, by any reasonable market model.

One of the best established stylized fact is the existence of volatility fluctuations that are long-ranged correlated in time. Empirical studies show that the volatility correlation function decays very slowly in time, and is well fitted by a power-law of the time lag with a small exponent 5, 6, 7, 8]. An exponential fit with a single time scale - suggested by several simple stochastic volatility (SV) models $9,10,11,12,13,14]$ - fails to describe the data: either the time scale is chosen to be small (a few days) and the long time tail is completely missed, or the time scale is chosen to be large (several hundreds of days), and the rapid initial decay is not properly accounted for. At least two time scales, and perhaps even three [15] are needed to reproduce the volatility dynamics. Recent multifractal models [8, 16, 17] actually postulate the existence of an infinity of time scales to build a self-similar volatility model with a power-law autocorrelation function.

Another, less well documented, effect is the negative return-volatility correlation: price drops are followed by an increased level of volatility. This effect was first noted by Black [18] and is usually called, somewhat inappropriatly, the 'leverage' effect, or asymmetric volatility correlation [19]. The presence of these correlations leads to skew in the distribution of returns and, correspondingly, to skew in the option smiles. The effect is much stronger for stock indices than for individual stocks, but appears to decay on rather short time scales (10-20 days [13, 20]), much shorter than the long time scale noted above for the volatility correlation.

Among the several SV models presented [1], both the Heston [10, 11, 14] and the Ornstein-Uhlenbeck 9, 12, 13] SV models are susceptible to contain an asymmetry parameter that generates some leverage correlations [21]. However,

\footnotetext{
* perello@ffn.ub.es

$\dagger$ jaume@ffn.ub.es

$\ddagger$ jean-philippe.bouchaud@science-finance.fr
} 
as mentioned above, these models contain a single time scale which governs both the volatility correlations and the return-volatility correlations, and cannot account for the different temporal behaviour of the two. Multifractal models, on the other hand, have only been very recently extended to consider the leverage effect [22, 23]. These models are however quite complex and analytical results for the unconditional and conditional return distribution over different time scales are only partially available [22, 24].

Our aim in this paper is to extend the exactly soluble Ornstein-Uhlenbeck SV model introduced in [9, 12, 13], such as to reproduce the multi-time scale dynamics reported above. In the present paper we allow for two different time scales, but the model can be generalised to account for as many time scales as needed, still retaining its exactly soluble nature. The basic idea is to make the mean reverting level of the volatility itself random and time dependent. The paper is divided into six sections. In section II we present our three-dimensional diffusion model. Sections III and IV respectively study the leverage and volatility correlations. In Section $\nabla$ we apply the model to actual data, thus providing a way of estimating the parameters of the model. Conclusions are drawn in section VI and some more technical details are relegated to appendices.

\section{A THREE-DIMENSIONAL DIFFUSION MODEL}

The dynamics of stock price $S(t)$ is usually modelled as a multiplicative diffusion process. It is commonly assumed that stock price $S(t)$ obeys the diffusion process:

$$
\frac{d S(t)}{S(t)}=\mu d t+\sigma d W_{1}(t)
$$

where $\mu$ is the drift, $\sigma$ is the so-called volatility and $W_{1}(t)$ is a Wiener process, i.e., $d W_{1}(t)=\xi_{1}(t) d t$, where $\xi_{1}(t)$ is Gaussian white noise with zero-mean and unit volatility:

$$
\left\langle\xi_{1}(t)\right\rangle=0 \quad \text { and } \quad\left\langle\xi_{1}(t) \xi_{1}\left(t^{\prime}\right)\right\rangle=\delta\left(t-t^{\prime}\right)
$$

For the sake of simplicity we will work with the zero-mean return process defined by

$$
d X(t) \equiv \frac{d S(t)}{S(t)}-\mu d t .
$$

Market dynamics is then described by a simpler stochastic differential equation (SDE)

$$
d X(t)=\sigma d W_{1}(t)
$$

As is well-known, this model does not satisfactorily explain many properties of the market. A possible improvement is to assume that, besides the return following the diffusion process (1), the volatility itself is a random variable, i.e., $\sigma=\sigma(t)$ is a stochastic process. This is done in the stochastic volatility models which appeared in the literature during late eighties 11], following similar discrete time 'ARCH' models. There are several ways to choose the random process describing the volatility [10, 11]. The simplest one consists in assuming that the volatility is also a diffusion process following the Ornstein-Uhlenbeck (OU) process [9]:

$$
d \sigma=-\alpha(\sigma-m) d t+k d W_{2}(t)
$$

where $\alpha>0, k>0$, and $m$ are nonrandom quantities, and $d W_{2}(t)$ is another Wiener process. The quantity $1 / \alpha$ is the characteristic time scale of the process and measures the strength of the reversion of $\sigma(t)$ to the constant $m$ which is the 'normal' level of volatility (see below). The quantity $k$ is the amplitude of the volatility fluctuations and it is sometimes called 'vol of vol'. As is well known, the stationary distribution of the OU process is Gaussian and this implies that the probability density does not vanish for $\sigma \rightarrow 0$. Since only $|\sigma|$ is meaningful, one has to 'fold' the negative part of the Gaussian onto the positive side with the result that the probability of $|\sigma|$ has a small hump for $|\sigma|$ small. This and the Gaussian shape of the distribution of the volatility are not very realistic because the empirical distribution of the volatility is rather found to be close to a log-normal, or to an inverse Gamma distribution [25, 26]. This is certainly a limitation of the present model. An interesting extension would be to consider the case where $\ln \sigma$, instead of $\sigma$, follows an OU process. Another limitation of the present model is the assumption that $d W_{1}(t)$ is Gaussian. Although volatility fluctuations indeed generate fat tails, the empirical frequency of very large returns (like crashes) cannot be reproduced within the class of Gaussian stochastic volatility models, and some extra 'jumps' must be allowed (see the empirical results and comments in Section V]. 
Two of us have recently studied the model represented by Eqs. (11)-(4) and showed 13] that it reproduces the leverage correlation only when the variations of volatility are anticorrelated with the variations of return. In other words, when both Wiener processes $W_{1}(t)$ and $W_{2}(t)$ appearing in Eqs. (11) and (4) are anticorrelated:

$$
\left\langle\xi_{1}(t) \xi_{2}\left(t^{\prime}\right)\right\rangle=\rho \delta\left(t-t^{\prime}\right)
$$

where $\rho<0$. As mentioned above the model given by Eqs. (11) and (4) together with Eq. (5) reproduces very well the observed leverage correlation but is unfortunately unable to reproduce simultaneously the long range volatility autocorrelation because leverage and volatility autocorrelation have both approximately the same characteristic time given by $1 / \alpha$.

In the volatility process given by Eq. (4), $m$ is a nonrandom constant and indicates the level to which the average volatility eventually converges as time increases [27]. In order to visualize this, suppose that the process begun in the infinite past $\left(t_{0} \rightarrow-\infty\right)$ which implies that at time $t$ the volatility has reached a stationary state, independent of initial conditions. In this situation one can easily see from the integration of Eq. (4) that

$$
\sigma(t)=m+k \int_{-\infty}^{t} e^{-\alpha\left(t-t^{\prime}\right)} d W_{2}\left(t^{\prime}\right) .
$$

From this we clearly see why $m$ can be called the normal level of volatility since (i) it is the stationary mean value of the volatility, $\langle\sigma(t)\rangle=m$, and (ii) $m$ is the value to which $\sigma(t)$ would return without any random innovation $(k=0)$.

However, there is no reason to believe that this 'normal' level of volatility is not itself slowly time evolving, with long periods where the average volatility is high (like stocks in the 30's), and other periods where it is smaller (like in the 60's). We will now relax this assumption and explore its consequences. We thus assume that $m=m(t)$ is a random process also obeying an OU stochastic differential equation:

$$
d m=-\alpha_{0}\left(m-m_{0}\right) d t+k_{0} d W_{3}(t),
$$

where $\alpha_{0}>0$ and $k_{0}>0$, and $m_{0}$ is now the 'true' long time average of the volatilty (but see the discussion in the conclusion). We therefore propose that the process describing the whole market dynamics is the following three-dimensional random process:

$$
\begin{aligned}
& d X(t)=\sigma(t) d W_{1}(t), \\
& d \sigma(t)=-\alpha[\sigma(t)-m(t)] d t+k d W_{2}(t), \\
& d m(t)=-\alpha_{0}\left[m(t)-m_{0}\right] d t+k_{0} d W_{3}(t),
\end{aligned}
$$

where $d W_{i}(t)=\xi_{i}(t) d t(i=1,2,3)$ are Wiener processes, i.e., $\xi_{i}(t)$ are zero-mean Gaussian white noises with crosscorrelation given by

$$
\left\langle\xi_{i}(t) \xi_{j}\left(t^{\prime}\right)\right\rangle=\rho_{i j} \delta\left(t-t^{\prime}\right)
$$

where $\rho_{i j}=\rho_{j i}$ and $\rho_{i i}=1$. We also suppose that $W_{3}(t)$ is independent of $W_{1}(t)$ and $W_{2}(t)$. Hence, the correlation matrix is

$$
\left(\rho_{i j}\right)=\left(\begin{array}{lll}
1 & \rho & 0 \\
\rho & 1 & 0 \\
0 & 0 & 1
\end{array}\right)
$$

where $-1 \leq \rho \leq 1$. As is common in finance, Eqs. (8)-(10) are interpreted in the Itô sense and for the rest of the paper we will follow the Itô convention [28]. A key consequence of the Itô convention is that $\sigma(t)$ and $m(t)$ are independent of any of the same time driving noises $d W_{i}(t)(i=1,2,3)$.

Note that the choice of the correlation matrix given by Eq. (12) assumes that the variations of return and instantaneous volatility are directly correlated while the variation of the volatility normal level is unaffected by recent price changes. Although reasonable from a financial point of view, there exists a different limit in which the normal level of volatility is directly correlated with the return. This case is treated in Appendix $\mathbf{C}$ where we focus on an alternative correlation matrix for which $\rho_{12}=0$ and $\rho_{13}=\rho$ instead of $\rho_{12}=\rho$ and $\rho_{13}=0$. We will see there that in this limit both the leverage and volatility correlations are governed, on long time scales, by the 'slow mode' of the model.

In what follows we will assume that at time $t$ the entire process has reached the stationary state. From Eq. (7) we see that the expression for $m(t)$ in the stationary state is

$$
m(t)=m_{0}+k_{0} \int_{-\infty}^{t} e^{-\alpha_{0}\left(t-t^{\prime}\right)} d W_{3}\left(t^{\prime}\right) .
$$


Now the stationary expression for $m(t)$ is given by the solution of Eq. (9) rather than by Eq. (6). We thus have

$$
\sigma(t)=\int_{-\infty}^{t}\left[k \xi_{2}\left(t^{\prime}\right)+\alpha m\left(t^{\prime}\right)\right] e^{-\alpha\left(t-t^{\prime}\right)} d t
$$

which, after using Eq. (13), reads

$$
\sigma(t)=m_{0}+k \int_{-\infty}^{t} e^{-\alpha\left(t-t^{\prime}\right)} d W_{2}\left(t^{\prime}\right)+\frac{k_{0}}{1-\lambda} \int_{-\infty}^{t}\left[e^{-\alpha_{0}\left(t-t^{\prime}\right)}-e^{-\alpha\left(t-t^{\prime}\right)}\right] d W_{3}\left(t^{\prime}\right),
$$

where

$$
\lambda=\frac{\alpha_{0}}{\alpha}
$$

We will see later on that empirical evidence implies that $0 \leq \lambda<1$, as suggested by the initial motivation of the model. One of the interest of this linear model is that one can also compute explicitely, using its Markovian nature the time evolution of the volatility conditioned to a given present value of the instantaneous volatility $\sigma\left(t_{0}\right)$ and of the 'normal' level of the volatility $m\left(t_{0}\right)$. This in turn opens the way to obtain the conditional distribution of returns over an arbitrary horizon $T$, a quantity needed for pricing options (see [9, 12, 13]).

Observe that since $m(t)$ is a Gaussian process its mean and variance read

$$
\langle m(t)\rangle=m_{0}, \quad \operatorname{Var}[m(t)]=m_{0}^{2} \nu_{0}^{2},
$$

and the autocorrelation is

$$
\langle m(t) m(t+\tau)\rangle=m_{0}^{2}\left(1+\nu_{0}^{2} e^{-\alpha_{0} \tau}\right) \quad(\tau \geq 0)
$$

where $\tau \geq 0$ and $\nu_{0}$ is defined by

$$
\nu_{0}^{2}=\frac{k_{0}^{2}}{2 m_{0}^{2} \alpha_{0}}
$$

Likewise $\sigma(t)$ is also Gaussian and has the following averages

$$
\langle\sigma(t)\rangle=m_{0}, \quad \operatorname{Var}[\sigma(t)]=m_{0}^{2}\left(\nu^{2}+\nu_{0}^{2} \frac{1}{1+\lambda}\right)
$$

and autocorrelation

$$
\langle\sigma(t) \sigma(t+\tau)\rangle=m_{0}^{2}\left[1+\left(\nu^{2}-\frac{\lambda \nu_{0}^{2}}{1-\lambda^{2}}\right) e^{-\alpha \tau}+\frac{\nu_{0}^{2}}{1-\lambda^{2}} e^{-\alpha_{0} \tau}\right]
$$

where $\tau \geq 0$, and

$$
\nu^{2}=\frac{k^{2}}{2 m_{0}^{2} \alpha} .
$$

\section{THE LEVERAGE EFFECT}

As recalled in the introduction, it has been known for long that volatility and returns are negatively correlated [18]. This correlation has however not been studied from a quantitative manner until quite recently [20], where the following 'leverage' correlation function was studied:

$$
\mathcal{L}(\tau) \equiv \frac{1}{Z}\left\langle d X(t+\tau)^{2} d X(t)\right\rangle
$$

where $d X(t)$ is the zero-mean return and

$$
Z=\left\langle d X(t)^{2}\right\rangle^{2}
$$


is a convenient normalization coefficient. A large amount of daily returns for both market indices and share prices were studied in [20], with the finding that,

$$
\mathcal{L}(\tau) \approx \begin{cases}-A e^{-\Gamma \tau}, & \text { if } \tau>0 \\ 0, & \text { if } \tau<0\end{cases}
$$

$(A, \Gamma>0)$. Hence, there is an exponentially decaying negative correlation between future volatility and past returns changes. No correlation is found between past volatility and future price changes (except perhaps for very short time lags $|\tau|$ ). In this way, a clear causality of the leverage effect is established, whereas there are conflicting claims on this issue in the literature [19]. For individual stocks, the coefficient $A$ is found to be close to 2, a value expected on the basis of simple 'retarded' effect, where the price changes are locally additive, rather than multiplicative random variables $[20]$. For indices, however, $A$ is much stronger $(A \approx 20)$, and the retarded interpretation fails. The coefficient $\Gamma$, on the other hand, defines the inverse relaxation time of the leverage correlation, and is found to be on the order of ten days for stock indices.

Let us study the leverage correlation within the framework of the three-dimensional diffusion model presented in Section II First, from Eq. (8), we calculate the correlation

$$
\left\langle d X(t) d X(t+\tau)^{2}\right\rangle=\left\langle\sigma(t) d W_{1}(t) \sigma(t+\tau)^{2} d W_{1}(t+\tau)^{2}\right\rangle
$$

Following the Itô convention, if $\tau \geq 0$ then $d W_{1}(t+\tau)$ is uncorrelated with the rest of stochastic variables. Thus, taking into account that

$$
\left\langle d W_{1}(t+\tau)^{2}\right\rangle=d t
$$

we have

$$
\left\langle d X(t) d X(t+\tau)^{2}\right\rangle=\left\langle\sigma(t) d W_{1}(t) \sigma(t+\tau)^{2}\right\rangle d t \quad(\tau>0)
$$

Otherwise, if $\tau \leq 0$, then $d W_{1}(t)$ is uncorrelated with the remaining variables. Hence, taking into account that $\left\langle d W_{1}(t)\right\rangle=0$, we get

$$
\left\langle d X(t) d X(t+\tau)^{2}\right\rangle=0 \quad(\tau \leq 0)
$$

Therefore,

$$
\left\langle d X(t) d X(t+\tau)^{2}\right\rangle=\left\langle\sigma(t) \sigma(t+\tau)^{2} d W_{1}(t)\right\rangle \mathrm{H}(\tau) d t
$$

where

$$
\mathrm{H}(\tau)= \begin{cases}1, & \text { if } \tau>0 \\ 0, & \text { if } \tau \leq 0\end{cases}
$$

is the Heaviside step function. Now the Novikov theorem allows us to write (see Appendix A

$$
\left\langle d X(t) d X(t+\tau)^{2}\right\rangle=2 \rho k e^{-\alpha \tau} \mathrm{H}(\tau)\langle\sigma(t) \sigma(t+\tau)\rangle d t^{2},
$$

where $\langle\sigma(t) \sigma(t+\tau)\rangle$ is given by Eq. (20).

On the other hand, as a consequence of the Itô convention, processes $\sigma(t)$ and $d W_{1}(t)$ are independent. Therefore from Eqs. (8) and (23) we see that

$$
Z=\left\langle d X(t)^{2}\right\rangle^{2}=\left\langle\sigma(t)^{2}\right\rangle^{2}\left\langle d W_{1}(t)^{2}\right\rangle^{2}=\left\langle\sigma(t)^{2}\right\rangle^{2} d t^{2}
$$

Taking into account Eq. (19), $Z$ reads

$$
Z=m_{0}^{4}\left(1+\nu^{2}+\frac{\nu_{0}^{2}}{1+\lambda}\right)^{2} d t^{2}
$$

Finally, the substitution of Eqs. (20), (28) and (29) into Eq. (22) yields

$$
\mathcal{L}(\tau)=-\mathrm{H}(\tau) A(\tau) e^{-\alpha \tau}
$$


where

$$
A(\tau) \equiv-\frac{2 \rho \nu \sqrt{2 \alpha}}{m_{0}(1+a+b)^{2}}\left[1+a e^{-\alpha \tau}+b e^{-\alpha_{0} \tau}\right]
$$

with:

$$
a=\nu^{2}-\frac{\lambda \hat{\nu}_{0}^{2}}{1-\lambda}, \quad b=\frac{\hat{\nu}_{0}^{2}}{1-\lambda},
$$

and

$$
\hat{\nu}_{0}^{2} \equiv \frac{\nu_{0}^{2}}{1+\lambda} .
$$

Note that Eq. (30) is not very different from Eq. (24) above: the dominant time decay is fixed by the short time scale of the volatility process $1 / \alpha$. As intuitively expected, the correlation coefficient $\rho$ between $d W_{1}$ (returns) and $d W_{2}$ (volatility changes) needs to be negative if we want to describe the observed anticorrelation.

\section{VOLATILITY CORRELATION}

The correlation for the volatility $\sigma(t)$ has been already given by Eq. (20). Nevertheless, in order to study the different time regimes appearing in the correlation, it is convenient to deal with a slightly different form of correlation. We will thus work with the function

$$
\operatorname{Corr}[\sigma(t), \sigma(t+\tau)]=\frac{\langle\langle\sigma(t) \sigma(t+\tau)\rangle\rangle}{\sqrt{\operatorname{Var}[\sigma(t)]} \sqrt{\operatorname{Var}[\sigma(t+\tau)]}}
$$

where $\langle\langle\sigma(t) \sigma(t+\tau)\rangle\rangle$ is the second cumulant of the volatility process defined by

$$
\langle\langle\sigma(t) \sigma(t+\tau)\rangle\rangle=\langle\sigma(t) \sigma(t+\tau)\rangle-\langle\sigma(t)\rangle\langle\sigma(t+\tau)\rangle .
$$

From Eqs (19) and (20) we have

$$
\operatorname{Corr}[\sigma(t), \sigma(t+\tau)]=M\left[a e^{-\alpha \tau}+b e^{-\alpha_{0} \tau}\right]
$$

where

$$
M=\frac{1}{\nu^{2}+\hat{\nu}_{0}^{2}}
$$

and $\hat{\nu}_{0}^{2}$ is given by Eq. (33).

If we assume that $\alpha_{0} \ll \alpha$ the volatility correlation (34) has two different regimes according to $\tau$ being small or large.

- Suppose that $\tau$ is small and such that the following two conditions meet simultaneously

$$
\alpha_{0} \tau \ll 1 \text { and } \alpha \tau \sim 1 .
$$

In this case, the characteristic time scale of volatility process given by $1 / \alpha$ (see Eq. (9) ) will dominate and the correlation reads

$$
\operatorname{Corr}[\sigma(t), \sigma(t+\tau)] \sim M\left(b+a e^{-\alpha \tau}\right), \quad\left(\alpha_{0} \tau \ll 1\right) .
$$

- Suppose now that $\tau$ is large and such that

$$
\alpha \tau \gg 1 \text { and } \quad \alpha_{0} \tau \sim 1 .
$$

In this case the characteristic time of the normal level of volatility $m(t)$ (see Eq. (10) is the dominant one and we see from Eq. (34) that

$$
\operatorname{Corr}[\sigma(t), \sigma(t+\tau)] \sim M b e^{-\alpha_{0} \tau}, \quad(\alpha \tau \gg 1) .
$$


Note that both approximations are consistent since we have assumed that $\alpha_{0} \ll \alpha$. This implies that $\lambda \ll 1$ (cf. Eq (15) ) is very small which, in turn, simplifies the form of the parameters $M, a$, and $b$. Indeed, now we can write

$$
M=\left(\nu^{2}+\nu_{0}^{2}\right)^{-1}+\mathrm{O}(\lambda)
$$

and

$$
a=\nu^{2}+\mathrm{O}(\lambda), \quad b=\nu_{0}^{2}+\mathrm{O}(\lambda) .
$$

Equations (36) and (37) show respectively the correlation behavior for small and large time $\tau$. The long range correlation for the volatility is governed by $\alpha_{0}$, that is, as expected, by the relaxation process of the volatility to its long time average $m_{0}$. This behavior is different from the leverage correlation which has a faster time decay $\alpha^{-1}$ that sets the 'fast' reversion of the volatility. Notice that this is consistent with empirical observations [2, 15, 27] (see also Fig. 11 and discussion in the next section).

At this stage, we should remind that the actual evaluation of the volatility $\sigma(t)$ is very difficult, since the volatility itself is not observed. In practice one can measure a noisy measure of the instantaneous volatility from:

$$
|\sigma(t)| \approx \sqrt{[X(t+\Delta t)-X(t)]^{2} / \Delta t}
$$

where $X(t)$ is the (undrifted) log-price defined above. Therefore, only $|\sigma(t)|$ can be extracted from data, and this is in principle insufficient to estimate (34). Another possible expression for the empirical autocorrelation of the volatility that is more directly observable is:

$$
\operatorname{Corr}\left[d X(t)^{2}, d X(t+\tau)^{2}\right]=\frac{1}{Y}\left\langle\left\langle d X(t)^{2} d X(t+\tau)^{2}\right\rangle\right\rangle
$$

where $Y^{2}=\operatorname{Var}\left[d X(t)^{2}\right] \operatorname{Var}\left[d X(t+\tau)^{2}\right]$ and

$$
\left\langle\left\langle d X(t)^{2} d X(t+\tau)^{2}\right\rangle\right\rangle=\left\langle d X(t)^{2} d X(t+\tau)^{2}\right\rangle-\left\langle d X(t)^{2}\right\rangle\left\langle d X(t+\tau)^{2}\right\rangle,
$$

is the second cumulant associated with $d X(t)^{2}$. This correlation function is particularly interesting because it is directly related to the kurtosis of the terminal distribution of price changes, a quantity which is in turn related to the curvature of volatility smiles [26].

In Appendix [B] we show that within the present Gaussian model, the empirical correlation coefficient given by Eq. (41) can be written in terms of the volatility autocorrelation as

$$
\operatorname{Corr}\left[d X(t)^{2}, d X(t+\tau)^{2}\right]=\frac{\langle\sigma(t) \sigma(t+\tau)\rangle^{2}-\langle\sigma(t)\rangle^{4}}{4\left\langle\sigma(t)^{2}\right\rangle^{2}-3\langle\sigma(t)\rangle^{4}},
$$

and from Eqs. (19) and (20) we explicitly write

$$
\operatorname{Corr}\left[d X(t)^{2}, d X(t+\tau)^{2}\right]=N\left[a\left(2+a e^{-\alpha \tau}\right) e^{-\alpha \tau}+b\left(2+b e^{-\alpha_{0} \tau}\right) e^{-\alpha_{0} \tau}+2 a b e^{-\left(\alpha+\alpha_{0}\right) \tau}\right]
$$

where $a$ and $b$ are given by Eq. (32) and

$$
N=\left[1+8\left(\nu^{2}+\hat{\nu}_{0}^{2}\right)+4\left(\nu^{2}+\hat{\nu}_{0}^{2}\right)^{2}\right]^{-1} .
$$

Observe that correlation (43) consists of a linear combination of decaying exponentials with arguments $\alpha_{0} \tau, \alpha \tau, 2 \alpha_{0} \tau$, $2 \alpha \tau$, and $\left(\alpha_{0}+\alpha\right) \tau$. We study some asymptotic limits for the correlation (43) as done above with $\sigma(t)$. We again consider the case when $\alpha_{0} \ll \alpha$ (i.e., $\left.\lambda \ll 1\right)$ and study the short and long $\tau$ behavior. For small $\tau$, when $\alpha_{0} \tau$ is small but $\alpha \tau$ is finite and not too small, we have

$$
\operatorname{Corr}\left[d X(t)^{2}, d X(t+\tau)^{2}\right] \approx N\left[(2+b) b+a\left(2+2 b+a e^{-\alpha \tau}\right) e^{-\alpha \tau}\right], \quad\left(\alpha_{0} \tau \ll 1\right) .
$$

In this regime, we only have exponentials with $\alpha$ as it happened with the sigma autocorrelation (c.f. Eq. (36)). For large $\tau$, the correlation is again dominated by the exponential with characteristic time scale $\alpha_{0}$ and reads

$$
\operatorname{Corr}\left[d X(t)^{2}, d X(t+\tau)^{2}\right] \approx N b\left(2+b e^{-\alpha_{0} \tau}\right) e^{-\alpha_{0} \tau} \quad(\alpha \tau \gg 1) .
$$

Observe that pairs of Eqs. (36)- (37) and Eqs. (45)- (46) contain the behavior that is empirically observed in financial markets. Although Eq. (43) has a much more complicated form, it still contains the two desired regimes for large and small $\tau$ as we have discussed above. 


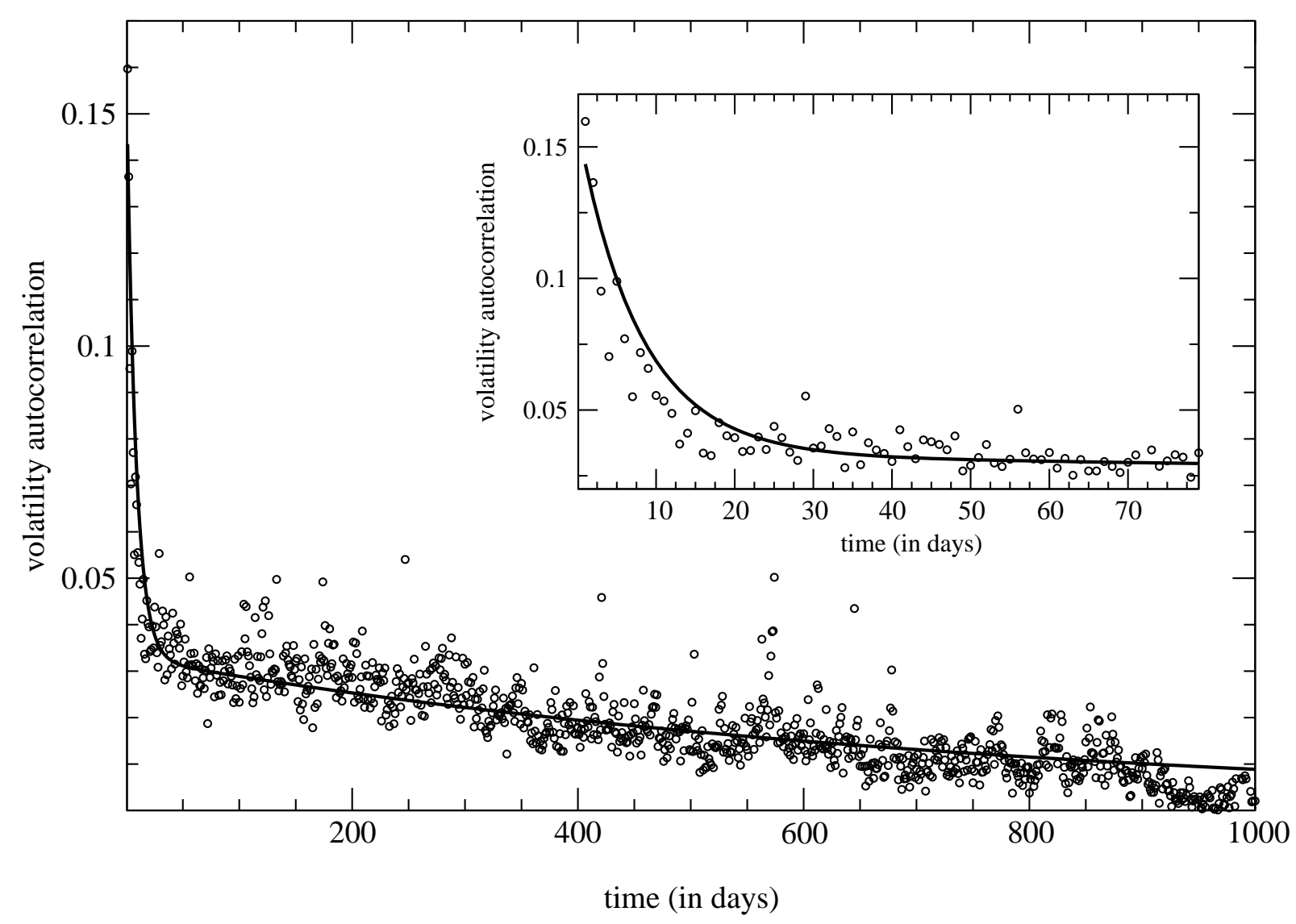

FIG. 1: The autocorrelation of $\Delta X(t)^{2}$ for the Dow-Jones daily index. We also plot their theoretical correlations given by Eq. (43) with the parameters estimated from the fit with $\alpha=0.1$ days $^{-1}, \alpha_{0}=1.3 \times 10^{-3}$ days $^{-1}, a=0.14$ and $b=0.04$. The inset is a zoom of the correlation focussing on the range from 1 to 80 days.

\section{ESTIMATION OF PARAMETERS}

Any acceptable market model must be easily calibrated to reproduce the dynamics of the market. In other words, the model should be supplemented by a systematic methodology for estimating its parameters. In our model we have six parameters to estimate. These parameters are $\rho, k$ and $k_{0}$ (or $\nu^{2}$ and $\left.\nu_{0}^{2}\right), \alpha$ and $\alpha_{0}$, and $m_{0}$ (see Eqs. (8) -(10)).

Let us discuss the way to estimate these parameters. We will proceed in a very similar way to the case given in Ref. 13 and perform the empirical estimation of the Dow-Jones index daily returns by approximating $d X$ by $\Delta X \equiv X(t+\Delta t)-X(t)$, i.e.

$$
d X(t) \approx X(t+\Delta t)-X(t),
$$

where $\Delta t=1$ day. From Eqs. (19) and (Bי) and taking into account that $\sigma(t)$ is independent of $d W_{1}(t)$, and that $d W_{1}(t)$ is Gaussian, we have

$$
\begin{aligned}
\operatorname{Var}[\Delta X] & =\left\langle\sigma(t)^{2}\right\rangle \Delta t=m_{0}^{2}\left(1+\nu^{2}+\hat{\nu}_{0}^{2}\right) \Delta t, \\
\operatorname{Var}\left[\Delta X^{2}\right] & =\left[3\left\langle\sigma(t)^{4}\right\rangle-\left\langle\sigma(t)^{2}\right\rangle\right] \Delta t^{2}=2 m_{0}^{4}\left[4\left(1+\nu^{2}+\hat{\nu}_{0}^{2}\right)^{2}-3\right] \Delta t^{2},
\end{aligned}
$$

where $\nu^{2}=k^{2} /\left(2 \alpha m_{0}^{2}\right)$ and $\hat{\nu}_{0}^{2}=k_{0}^{2} /\left(2 \alpha_{0} m_{0}^{2}(1+\lambda)\right)$. From these equations we get

$$
\frac{1}{\left(1+\nu^{2}+\hat{\nu}_{0}^{2}\right)^{2}}=\frac{4}{3}-\frac{1}{6} \frac{\operatorname{Var}\left[\Delta X^{2}\right]}{\operatorname{Var}[\Delta X]^{2}}
$$

Hence, we can estimate the values of $\left(\nu^{2}+\hat{\nu}_{0}^{2}\right)$ once we know the empirical values of the daily variances of $\Delta X$ and $\Delta X^{2}$. In a second step, $m_{0}$ is estimated from the knowledge of $\left(\nu^{2}+\hat{\nu}_{0}^{2}\right)$ and the empirical value of $\operatorname{Var}[\Delta X]$. In Table \we briefly report these operations and give the corresponding estimation of $\left(\nu^{2}+\hat{\nu}_{0}^{2}\right)$ and $m_{0}$ for the Dow-Jones index time-series from 1900 until 2000. 


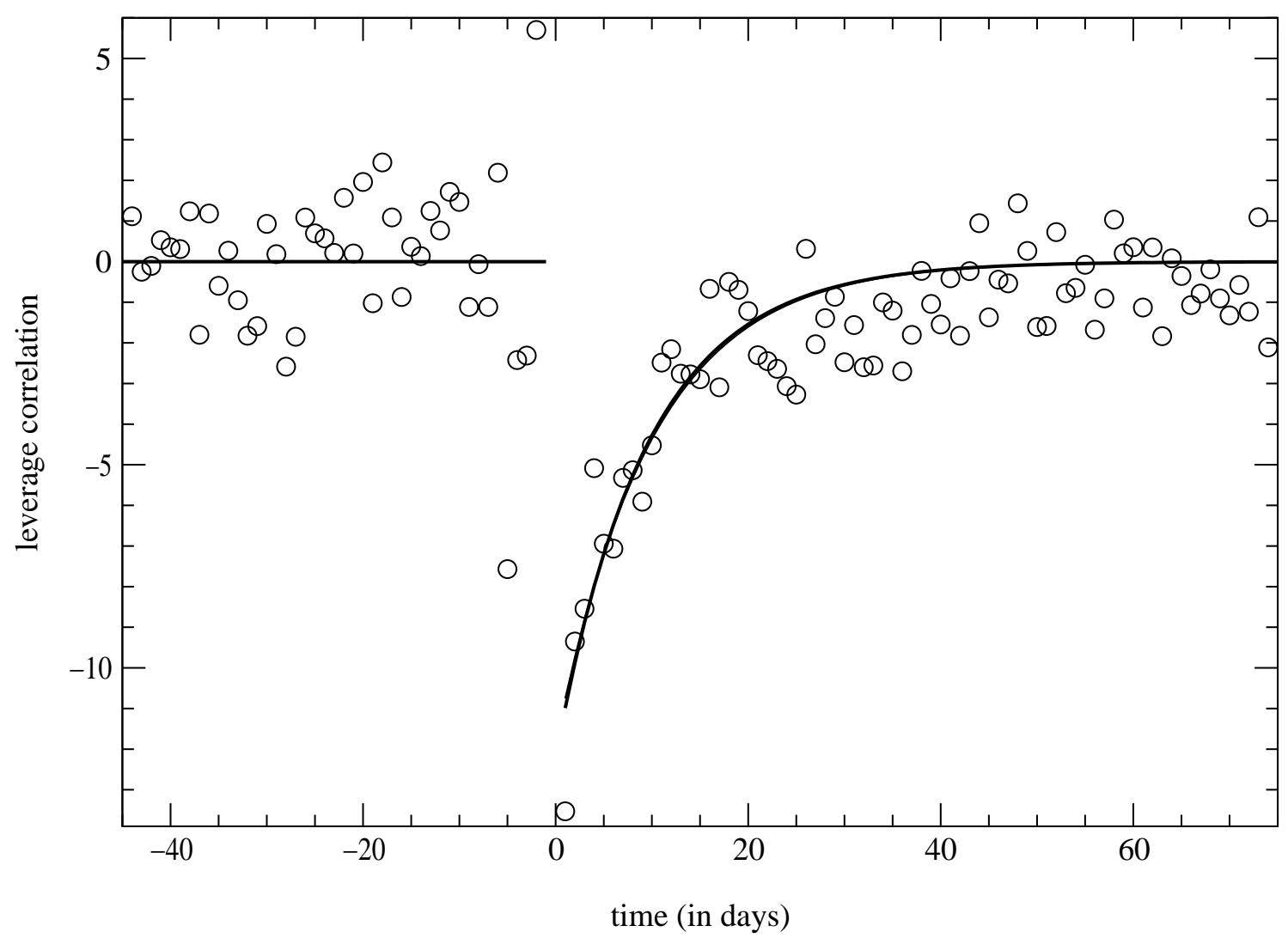

FIG. 2: The leverage effect in the Dow-Jones daily index. We plot the leverage function $\mathcal{L}(\tau)$ for the Dow-Jones index from 1900 until 2000. We also plot a fit with the leverage function (30) with $\alpha=0.1$ days $^{-1}, a=0.14, b=0.04$, and $m=1.19 \times 10^{-2}$ days $^{-1 / 2}$ finding that $\rho=-0.48$ (see Table II).

TABLE I: Estimate from return variances. We estimate parameters of the model from Dow-Jones historical daily returns from 1900 to 2000 . We use the identity (48) and take the variance (47) to derive the estimated quantities $\left(\nu^{2}+\hat{\nu}_{0}^{2}\right)$ and $m_{0}$.

\begin{tabular}{ccc}
\hline \hline & & \\
Estimators & Dow-Jones daily return & Theoretical values \\
\hline & & \\
$\operatorname{Var}[\Delta X(t)]$ & $1.68 \times 10^{-4}$ & $m_{0}^{2}\left(1+\nu^{2}+\hat{\nu}_{0}^{2}\right) \Delta t$ \\
$\operatorname{Var}\left[\Delta X(t)^{2}\right]$ & $10.5 \times 10^{-8}$ & $2 m_{0}^{4}\left[4\left(\nu^{2}+\hat{\nu}_{0}^{2}\right)^{2}-3\right] \Delta t^{2}$ \\
& \\
$\begin{array}{c}\text { Parameter estimates } \\
\left(\nu^{2}+\hat{\nu}_{0}^{2}\right)\end{array}$ & 0.18 \\
$m_{0}$ & $18.9 \%$ year $^{-1 / 2}$ \\
\end{tabular}

Since $\left(\nu^{2}+\hat{\nu}_{0}^{2}\right)$ is always positive and $\left(1+\nu^{2}+\hat{\nu}_{0}^{2}\right)^{2} \geq 1$, we see from Eq. (48) that

$$
2 \leq \frac{\operatorname{Var}\left[\Delta X^{2}\right]}{\operatorname{Var}[\Delta X]^{2}}<8,
$$

and the kurtosis defined by

$$
\kappa=\frac{\operatorname{Var}\left[\Delta X^{2}\right]}{\operatorname{Var}[\Delta X]^{2}}-2
$$


TABLE II: Parameter estimation calculated from the volatility autocorrelation (43) and the leverage correlation (30). We estimate $\alpha, \hat{\nu}_{0}^{2}$, and $\nu^{2}$ from the empirical volatility autocorrelation of Dow-Jones stock index plotted in Fig. 1 We estimate the parameters $\rho$ and check $\alpha$ from the fit of the Dow-Jones stock index data leverage plotted in Fig. 2

\begin{tabular}{lr}
\hline \hline & \\
Estimators & Dow-Jones data estimation \\
\hline \multicolumn{2}{l}{ Volatility autocorrelation } \\
$\alpha$ & 0.1 days $^{-1}$ \\
$\alpha_{0}$ & $1.3 \times 10^{-3}$ days $^{-1}$ \\
$a \simeq \nu^{2}$ & 0.14 \\
$b \simeq \nu_{0}^{2}$ & 0.04 \\
$\lambda=\alpha_{0} / \alpha$ & $1.3 \times 10^{-2}$ \\
$k=m_{0} \sqrt{2 \alpha \nu^{2}}$ & $2.0 \times 10^{-3}$ days $^{-1}$ \\
$k_{0}=m_{0} \sqrt{2 \alpha_{0} \nu_{0}^{2}}$ & $1.2 \times 10^{-4}$ days $^{-1}$ \\
& \\
Leverage & \\
$\mathcal{L}\left(0^{+}\right)$ & -11.9 \\
$\rho$ & \\
\hline \hline
\end{tabular}

has the bounds

$$
0 \leq \kappa<6
$$

As expected, volatility fluctuations induce some excess kurtosis, but this excess kurtosis cannot take arbitrarily high values. For the Dow-Jones index data and after taking away 'crash days' of amplitude greater than $12 \%$, we get a kurtosis of $\kappa \approx 1.7$ which is consistent with the requirement (49). The removal of these outliers only represents 11 data points, a very small fraction of the total amount (more than 27000 sample data points). However, as many authors have reported [29, 30, 31], the total kurtosis is found to be larger, perhaps even infinite (in particular for higher - intraday - frequencies). This is a limitation of the present model for describing higher frequency movements where one should eventually take into account a non Gaussian character for $d W_{1}$ (see the discussion in 26] and Refs. [32, 33])

We have four more parameters to estimate, $\alpha, \alpha_{0}, \nu^{2}$ and $\rho$, and we will use both the leverage and the volatility correlations to obtain them. As we see in Figs. 1] and 2 the leverage correlation appears to be much more noisy than the volatility autocorrelation. For this reason we start with volatility autocorrelation for quantifying $\alpha, \alpha_{0}, \nu^{2}$. We subsequently analyze the leverage correlation for testing the estimated $\alpha$ and for finally obtaining the parameter $\rho$.

As we have shown in the Section IV there are different ways to interpret the volatility correlation from empirical data. In the previous Section our choice was to take $\Delta X(t)^{2}$ as the square of the daily volatility. We have computed the empirical volatility correlation and plotted it in Fig. 1 with the corresponding fit with our theoretical correlation in Eq. (43):

$$
\operatorname{Corr}\left[d X(t)^{2}, d X(t+\tau)^{2}\right]=N\left[a\left(2+a e^{-\alpha \tau}\right) e^{-\alpha \tau}+b\left(2+b e^{-\alpha_{0} \tau}\right) e^{-\alpha_{0} \tau}+2 a b e^{-\left(\alpha+\alpha_{0}\right) \tau}\right],
$$

where $a=\nu^{2}-\lambda \hat{\nu}_{0}^{2} /(1-\lambda), b=\hat{\nu}_{0}^{2} /(1-\lambda), N=\left[1+8\left(\nu^{2}+\hat{\nu}_{0}^{2}\right)+4\left(\nu^{2}+\hat{\nu}_{0}^{2}\right)^{2}\right]^{-1}$. The fit plotted in Fig. 11 is done with the constraint that $a+b=\left(\nu^{2}+\hat{\nu}_{0}^{2}\right)=0.18$.

The proposed fit is obtained by taking $1 / \alpha_{0} \simeq 3$ years and $1 / \alpha \simeq 10$ days. Recall that $\lambda=\alpha_{0} / \alpha$ is been assumed in previous Sections to be very small and this is now confirmed by empirical observations since $\lambda=1.3 \times 10^{-2}$. In such a case, we can consider that $a \simeq \nu^{2}$ and $b \simeq \nu_{0}^{2}$. The results obtained are given in Table jointly with $k$ and $k_{0}$ that are given by Eqs. (18) and (21).

The leverage effect provides a way to estimate the correlation coefficient $\rho$. It also serves us as a test of consistency in the estimation of the characteristic time $1 / \alpha$. In a previous work [13], it is been suggested that $1 / \alpha \simeq 20$ days. 

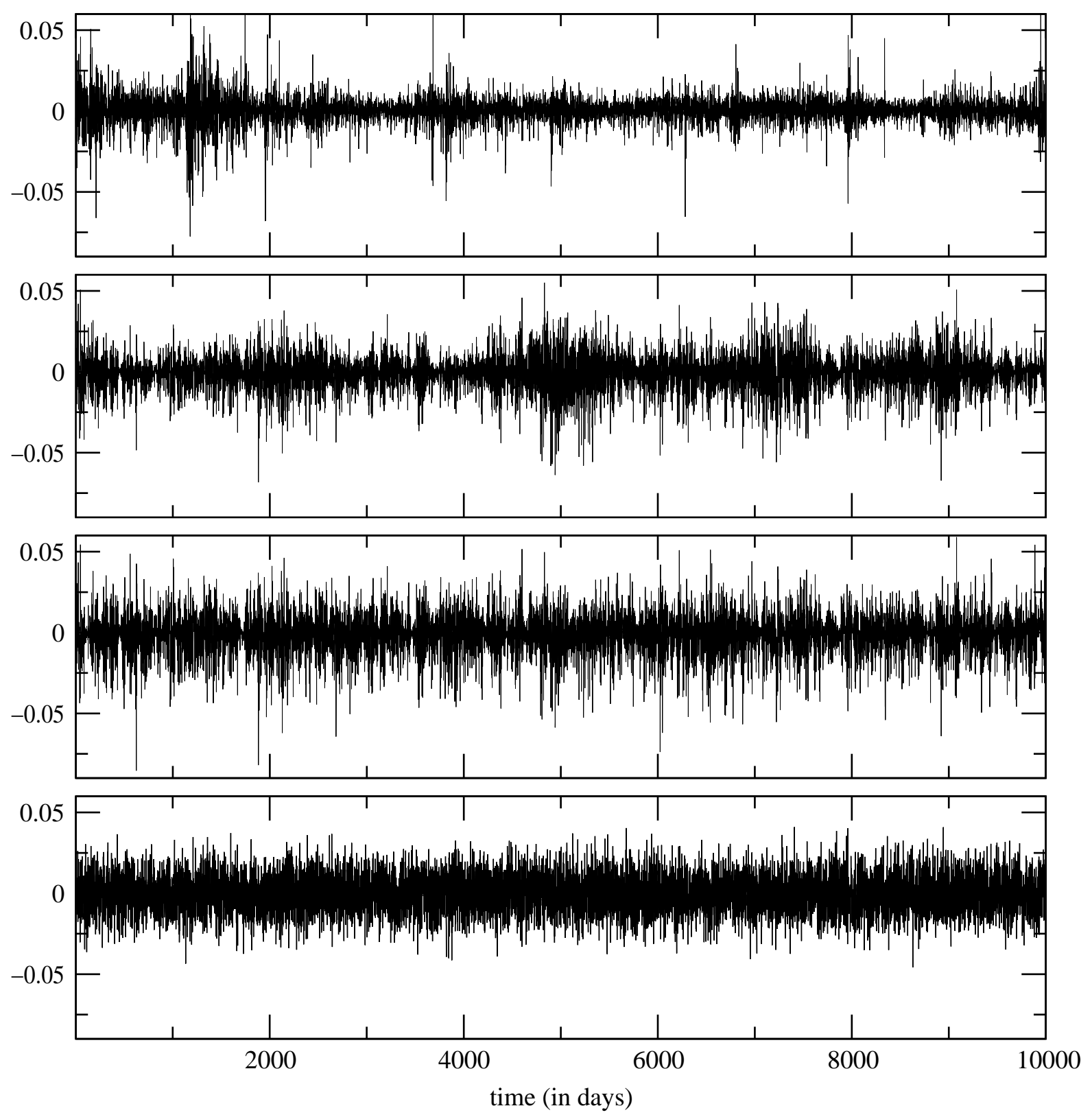

FIG. 3: Dow-Jones index and simulation paths over 10000 days which corresponds to approximately 40 years. Top figure shows the Dow-Jones index daily return changes over 10000 trading days and compare it with several simulations. From top to bottom we show random path of our three-dimensional stochstic volatility model (8)-(10), the path of the two-dimensional approach (3) (4) and the Wiener process (3) assuming constant $\sigma$. Parameters are given in Tables 【and II

However, the fit of the leverage function (30)-(31) to the Dow-Jones daily data (Fig. 22) is not very binding. The quality of this non linear fit remains basically unchanged for modifications in $1 / \alpha$ inside the range $7-20$ days. For this reason we will keep our estimated $\alpha$ from volatility autocorrelation and accept that the results from the leverage correlation are consistent with the ones derived from the volatility correlation.

Let us now focus on the estimation of $\rho$. Recall that our theoretical leverage is given by Eqs. (30)-(31) with $a$ and $b$ given by Eq. (32). When $\tau \rightarrow 0^{+}$, we have

$$
\mathcal{L}\left(0^{+}\right)=\frac{2 \rho \nu \sqrt{2 \alpha}}{\left(1+\nu^{2}+\hat{\nu}_{0}^{2}\right) m}
$$

which allows us to estimate $\rho$ once we know $\alpha=0.1$ days $^{-1}$ and $\left(\nu^{2}+\hat{\nu}_{0}^{2}\right)=0.18$. All these operations are summarized in Table III The estimated magnitudes $\mathcal{L}\left(0^{+}\right)$and $\alpha$ obtained from the Dow-Jones index are of the same order as 


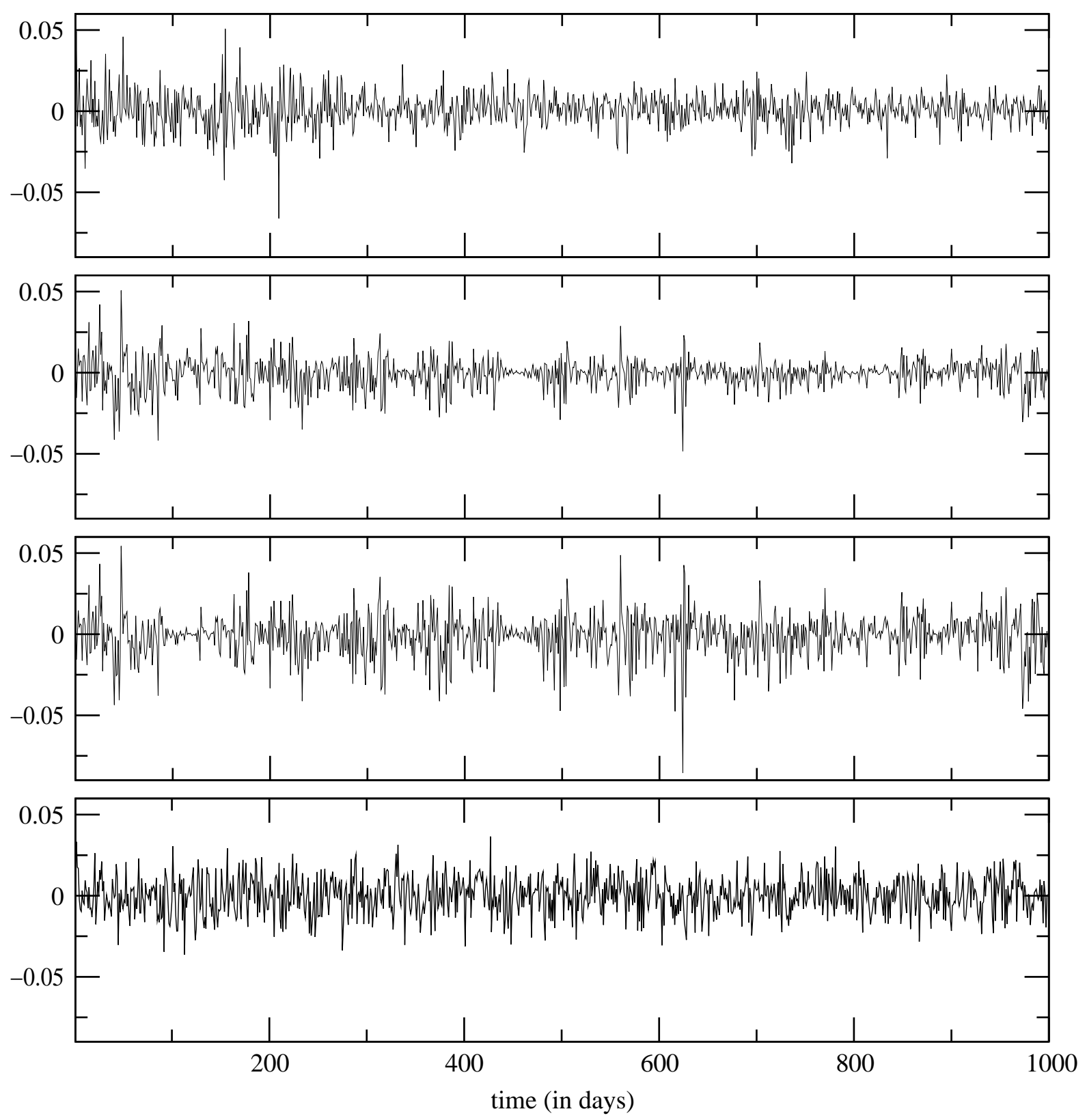

FIG. 4: Dow-Jones index and simulation paths over 1000 days (approximately 4 years). Top figure shows the Dow-Jones index daily return changes over 1000 trading days and compare it with several simulations. From top to bottom we show random path of our three-dimensional stochstic volatility model (8)-(10), the path of the two-dimensional approach (3)-(4) and the Wiener process (3) assuming constant $\sigma$. Parameters are given in Tables \and

those given in [20] from an average over several stock indices. The value of $\alpha$ is also consistent with that found by Dragulescu and Yakovenko within the Heston model [14] (for which the volatility correlation function is a single exponential).

Finally, in Figs. 3 and 4 we simulate the resulting process with the parameters estimated above. Once we know the six estimated parameters, we can simulate the random dynamics for $\Delta X(t)$ and compare it with the empirical DowJones time series. We also simulate the ordinary geometric Brownian motion where the volatility is constant together with the two-dimensional SV model studied in 13] in which the normal level of volatility is constant. In Figs. 3] and 4 we see that the ordinary geometric Brownian model cannot describe both the largest and the smallest fluctuations of daily returns. Furthermore, the two-dimensional SV model is not able to describe the clustering that appears when we take a longer number of days (see Fig. 31). Hence, we can conclude that SV models generate trajectories similar to 
that of the Dow-Jones but the three-dimensional model presented herein improves this description when we observe a wider time window. On the other hand, one should keep in mind that some aspects of the empirical data, such as the nearly log-normal distribution of volatility, or the extreme tails of the distribution of returns, are not reproduced by the present version of the model.

\section{CONCLUSIONS}

In this paper, we have suggested a simple way to build a stochastic volatility model that accounts for the presence of multiple time scales in financial markets. The volatility correlation function contains at least a 'short' time scale (tens of days) and a very long time scale that does not seem to be present in the asymmetric return-volatility, which only exhibits the 'short' time scale. The idea is to let the 'normal' level of volatility be itself time dependent and evolve over long time scales. The simplest setting is that of the linear Ornstein-Uhlenbeck model, where the calculations are simple [9, 12, 13], and which can easily be calibrated to market data. The overall description of the volatility and the leverage correlation using this three dimensional model is quite satisfactory, although several deficiencies remain: (a) the volatility process appears to need at least a third, very short time scale (on the order of a day), to be satisfactorily accounted for, (b) the empirical distribution of volatility is poorly reproduced by the present Gaussian model, and (c) the assumption that $d W_{1}$ is Gaussian does not allow one to account for extreme events. There are several natural extensions of the present model that could deal with these discrepancies: (i) one could generalize the Heston model as is done here, such as to avoid the unrealistic 'hump' of the volatility distribution close to zero; (ii) one could consider a model where the log-volatility, rather than the volatility, obeys an Ornstein-Uhlenbeck process, and (iii) one could build a 'Matrioshka doll' Ornstein-Uhlenbeck process where the construction given here is iterated as many times as needed to reproduce all the empirical time scales. In the limit of an infinite number of time scales, it might be possible to construct in an alternative way the multifractal random walk of Bacry, Muzy and Delour. Finally, it would be useful to extend the computation of the present paper to compute the conditional distribution of price changes over different time scales, such as to obtain option prices. We hope to report the results of this computation in the near future.

\section{Acknowledgments}

This work has been supported in part by Dirección General de Investigación under contract No. BFM2000-0795 and by Generalitat de Catalunya under contract No. 2000 SGR-00023. J-P B wants to thank M. Potters, A. Matacz, F. Selmi and V. Yakovenko for several useful discussions.

\section{APPENDIX A: THE NOVIKOV THEOREM}

The Novikov theorem provides a way of evaluating averages involving the Wiener process and functionals of it. Suppose we have a well-behaved function of time $f(t ;[\boldsymbol{\xi}])$ also depending functionally on an $n$-dimensional Gaussian white noise $\boldsymbol{\xi}(t)=\left(\xi_{1}(t), \cdots, \xi_{n}(t)\right)$. The Novikov theorem (see Ref. 34 for further details) states that the nonlinear average $\left\langle f(t ;[\boldsymbol{\xi}]) \xi_{j}\left(t^{\prime}\right)\right\rangle$ can be written as

$$
\left\langle f(t ;[\boldsymbol{\xi}]) \xi_{j}\left(t^{\prime}\right)\right\rangle=\left\langle\frac{\delta f(t ;[\boldsymbol{\xi}])}{\delta \xi_{j}\left(t^{\prime}\right)}\right\rangle, \quad(j=1,2,3, \cdots),
$$

where $\delta f(t ;[\boldsymbol{\xi}]) / \delta \xi_{j}\left(t^{\prime}\right)$ stands for the functional derivative of $f$ with respect to $\boldsymbol{\xi}[35]$. We can write an alternative, and sometimes more convenient form of the theorem if we specify the correlation between the $n$ components of $\boldsymbol{\xi}(t)$ :

$$
\left\langle\xi_{i}\left(t_{1}\right) \xi_{j}\left(t_{2}\right)\right\rangle=\rho_{i j} \delta\left(t_{1}-t_{2}\right) .
$$

One can easily see that in this case we can write

$$
\xi_{i}(t)=\xi(t)+\sum_{j \neq i} \rho_{i j} \xi_{j}(t),
$$

where $\xi(t)$ is a Gaussian white noise independent of any other component of $\boldsymbol{\xi}(t)$, i.e., $\left\langle\xi(t) \xi_{j}\left(t^{\prime}\right)\right\rangle=0$ for any $j \neq i$ and $t^{\prime}$. On the other hand the chain rule applied to the functional derivative allows us to write [35]

$$
\left\langle\frac{\delta f(t ;[\boldsymbol{\xi}])}{\delta \xi_{j}\left(t^{\prime}\right)}\right\rangle=\int d t^{\prime \prime} \sum_{i=1}^{n}\left\langle\frac{\delta f(t ;[\boldsymbol{\xi}])}{\delta \xi_{i}\left(t^{\prime \prime}\right)} \frac{\partial \xi_{i}\left(t^{\prime \prime}\right)}{\partial \xi_{j}\left(t^{\prime}\right)}\right\rangle .
$$


Then, taking into account Eq. (A2), the Novikov theorem can be set in the following alternative form:

$$
\left\langle f(t ;[\boldsymbol{\xi}]) \xi_{j}\left(t^{\prime}\right)\right\rangle=\sum_{i=1}^{n} \rho_{i j}\left\langle\frac{\delta f(t ;[\boldsymbol{\xi}])}{\delta \xi_{i}\left(t^{\prime}\right)}\right\rangle .
$$

We are now able to prove Eq. (28). In this case we have to consider the average $\left\langle\sigma(t) \sigma(t+\tau)^{2} \xi_{1}(t)\right\rangle$. Consequently $f(t ;[\boldsymbol{\xi}])=\sigma(t) \sigma(t+\tau)^{2}$ and the Novikov theorem, in the form given by Eq. (A1), tell us that

$$
\left\langle\sigma(t) \sigma(t+\tau)^{2} \xi_{1}(t)\right\rangle=\left\langle\frac{\delta\left[\sigma(t) \sigma(t+\tau)^{2}\right]}{\delta \xi_{1}(t)}\right\rangle .
$$

On the other hand we know from Eq. (14) that

$$
\sigma(t)=m_{0}+k \int_{-\infty}^{t} \xi_{2}\left(t^{\prime}\right) e^{-\alpha\left(t-t^{\prime}\right)} d t^{\prime}+\frac{k_{0}}{1-\lambda} \int_{-\infty}^{t} \xi_{3}\left(t^{\prime}\right)\left[e^{-\alpha_{0}\left(t-t^{\prime}\right)}-e^{-\alpha\left(t-t^{\prime}\right)}\right] d t^{\prime},
$$

where the integrals are represented in the Itô sense. Observe that $\sigma(t)$ depends functionally on $\xi_{2}(t)$ and $\xi_{3}(t)$ but not on $\xi_{1}(t)$ and, as it is shown by Eq. (12), $\xi_{1}(t)$ and $\xi_{2}(t)$ are the only two correlated process with correlation coefficient $\rho$. Thus, the functional derivative reads

$$
\frac{\delta\left[\sigma(t) \sigma(t+\tau)^{2}\right]}{\delta \xi_{1}(t)}=\rho \frac{\delta\left[\sigma(t) \sigma(t+\tau)^{2}\right]}{\delta \xi_{2}(t)},
$$

where we have used Eq. A2 to see that $\partial \xi_{2}(t) / \partial \xi(t)_{1}=\rho$. Therefore,

$$
\frac{\delta\left[\sigma(t) \sigma(t+\tau)^{2}\right]}{\delta \xi_{1}(t)}=\rho\left[\sigma(t+\tau)^{2} \frac{\delta \sigma(t)}{\delta \xi_{2}(t)}+2 \sigma(t) \sigma(t+\tau) \frac{\delta \sigma(t+\tau)}{\delta \xi_{2}(t)}\right] .
$$

The functional derivatives of $\sigma(t)$ and $\sigma(t+\tau)$ with respect to $\xi_{2}(t)$ can be obtained through Eq. A5 after taking into account that $\xi_{3}(t)$ is independent of $\xi_{2}(t)$ and that $\delta \xi_{2}\left(t^{\prime}\right) / \delta \xi_{2}(t)=\delta\left(t-t^{\prime}\right)$ [35]. Thus

$$
\frac{\delta \sigma(t)}{\delta \xi_{2}(t)}=k \mathrm{H}(0)=0
$$

since $H(0)=0(c f$. Eq. (27) $)$. Note that this is consistent with the Itô interpretation which assumes that $\xi_{2}(t)$ lies outside of the integration interval $(-\infty, t)$ of the first integral on the rhs of Eq. A5). Analogously

$$
\frac{\delta \sigma(t+\tau)}{\delta \xi_{2}(t)}=k e^{-\alpha \tau} \mathrm{H}(\tau)
$$

Finally, from Eq (A6), we have

$$
\left\langle\sigma(t) \sigma(t+\tau)^{2} \xi_{1}(t)\right\rangle=2 \rho k e^{-\alpha \tau}\langle\sigma(t) \sigma(t+\tau)\rangle \mathrm{H}(\tau)
$$

which proves Eq. (28).

\section{APPENDIX B: DERIVATION OF EQUATION (42)}

We will demonstrate the equality

$$
\frac{\left\langle d X(t)^{2} d X(t+\tau)^{2}\right\rangle-\left\langle d X(t)^{2}\right\rangle\left\langle d X(t+\tau)^{2}\right\rangle}{\sqrt{\operatorname{Var}\left[d X(t)^{2}\right]} \sqrt{\operatorname{Var}\left[d X(t+\tau)^{2}\right]}}=\frac{\langle\sigma(t) \sigma(t+\tau)\rangle^{2}-\langle\sigma(t)\rangle^{4}}{4\left\langle\sigma(t)^{2}\right\rangle^{2}-3\langle\sigma(t)\rangle^{4}},
$$

which is equivalent to prove Eq. (42) from Eq. (41). To this end we will deal with each term on the left hand side of Eq. (B1) separately.

We first derive $\left\langle d X(t)^{2} d X(t+\tau)^{2}\right\rangle$. From Eq. (3) we have

$$
\left\langle d X(t)^{2} d X(t+\tau)^{2}\right\rangle=\left\langle\sigma(t)^{2} \sigma(t+\tau)^{2} d W_{1}(t) \xi_{1}(t)\right\rangle d t^{2}
$$


where we have assumed that $d W_{1}(t+\tau)$ is independent of the rest of stochastic variables and also that $\left\langle d W_{1}(t+\tau)^{2}\right\rangle=$ $d t$. Since $d W_{1}(t)=\xi_{1}(t) d t$ we can write the average on the rhs of Eq. (B2) as $\left\langle\sigma(t)^{2} \sigma(t+\tau)^{2} d W_{1}(t) \xi_{1}(t)\right\rangle$. Now the Novikov theorem, Eqs. A1 and (A3), yields

$$
\begin{array}{r}
\left\langle\sigma(t)^{2} \sigma(t+\tau)^{2} d W_{1}(t) \xi_{1}(t)\right\rangle=2 \rho\left[\left\langle\frac{\delta \sigma(t)}{\delta \xi_{2}(t)} \sigma(t) \sigma(t+\tau)^{2} d W_{1}(t)\right\rangle+\left\langle\sigma(t)^{2} \sigma(t+\tau) \frac{\delta \sigma(t+\tau)}{\delta \xi_{2}(t)} d W_{1}(t)\right\rangle\right] \\
+\left\langle\sigma(t)^{2} \sigma(t+\tau)^{2} \frac{\delta\left[d W_{1}(t)\right]}{\delta \xi_{1}(t)}\right\rangle
\end{array}
$$

which, after using Eqs. (A7)- (A8), reads

$$
\left\langle\sigma(t)^{2} \sigma(t+\tau)^{2} d W_{1}(t) \xi_{1}(t)\right\rangle=2 \rho k e^{-\alpha \tau} \mathrm{H}(\tau)\left\langle\sigma(t)^{2} \sigma(t+\tau) d W_{1}(t)\right\rangle+\left\langle\sigma(t)^{2} \sigma(t+\tau)^{2} \frac{\delta\left[d W_{1}(t)\right]}{\delta \xi_{1}(t)}\right\rangle .
$$

In order to obtain an expression for the functional derivative $\delta\left[d W_{1}(t)\right] / \delta \xi_{1}(t)$, we write $d W_{1}(t)$ in a somewhat intricate form

$$
d W_{1}(t)=\int_{t}^{t+d t} \xi_{1}\left(t^{\prime}\right) d t^{\prime}
$$

Thus

$$
\frac{\delta\left[d W_{1}(t)\right]}{\delta \xi_{1}(t)}=\int_{t}^{t+d t} \delta\left(t-t^{\prime}\right) d t^{\prime}=1
$$

Hence

$$
\left\langle\sigma(t)^{2} \sigma(t+\tau)^{2} d W_{1}(t) \xi_{1}(t)\right\rangle=2 \rho k e^{-\alpha \tau} \mathrm{H}(\tau)\left\langle\sigma(t) \sigma(t+\tau)^{2} d W_{1}(t)\right\rangle+\left\langle\sigma(t)^{2} \sigma(t+\tau)^{2}\right\rangle .
$$

We apply again the Novikov theorem to the average $\left\langle\sigma(t) \sigma(t+\tau)^{2} d W_{1}(t)\right\rangle$ with the result

$$
\left\langle\sigma(t) \sigma(t+\tau)^{2} d W_{1}(t)\right\rangle=\rho\left\langle\frac{\delta}{\delta \xi_{2}(t)}\left[\sigma(t)^{2} \sigma(t+\tau)\right]\right\rangle d t,
$$

which, after using Eqs. (A7)- (A8), yields

$$
\left\langle\sigma(t)^{2} \sigma(t+\tau) d W_{1}(t)\right\rangle=\rho k e^{-\alpha \tau} \mathrm{H}(\tau)\left\langle\sigma(t)^{2}\right\rangle d t .
$$

Substituting this into Eq. (B33) we have

$$
\left\langle\sigma(t)^{2} \sigma(t+\tau)^{2} d W_{1}(t) \xi_{1}(t)\right\rangle=2 \rho^{2} k^{2} e^{-2 \alpha \tau} \mathrm{H}(\tau)\left\langle\sigma(t)^{2}\right\rangle d t+\left\langle\sigma(t)^{2} \sigma(t+\tau)^{2}\right\rangle .
$$

Notice that due to the differential $d t$, the first term on the rhs of this equation is negligible in front of the second term. Thus

$$
\left\langle\sigma(t)^{2} \sigma(t+\tau)^{2} d W_{1}(t) \xi_{1}(t)\right\rangle=\left\langle\sigma(t)^{2} \sigma(t+\tau)^{2}\right\rangle+\mathrm{O}(d t),
$$

and the substitution of Eq. (B4) into Eq. (B2) yields

$$
\left\langle d X(t)^{2} d X(t+\tau)^{2}\right\rangle=\left[\left\langle\sigma(t)^{2} \sigma(t+\tau)^{2}\right\rangle+\mathrm{O}(d t)\right] d t^{2} .
$$

On the other hand, since $d W_{1}(t)$ is independent of $\sigma(t)$ we can write

$$
\left\langle d X(t)^{2}\right\rangle\left\langle d X(t+\tau)^{2}\right\rangle=\left\langle\sigma(t)^{2}\right\rangle\left\langle\sigma(t+\tau)^{2}\right\rangle d t^{2}
$$

and, taking into account that process is in the stationary regime, we have

$$
\left\langle d X(t)^{2}\right\rangle\left\langle d X(t+\tau)^{2}\right\rangle=\left\langle\sigma(t)^{2}\right\rangle^{2} d t^{2} .
$$

Finally, we derive the variance:

$$
\operatorname{Var}\left[d X(t)^{2}\right]=\left\langle\sigma(t)^{4}\right\rangle\left\langle d W(t)^{4}\right\rangle-\left\langle\sigma(t)^{2}\right\rangle^{2}\left\langle d W(t)^{2}\right\rangle^{2},
$$


where we take into account that $\sigma(t)$ and $d W_{1}(t)$ are independent. We can go further since $\left\langle d W(t)^{4}\right\rangle=3\left\langle d W(t)^{2}\right\rangle^{2}=$ $3 d t^{2}$. Hence,

$$
\operatorname{Var}\left[d X(t)^{2}\right]=3\left\langle\sigma(t)^{4}\right\rangle d t^{2}-\left\langle\sigma(t)^{2}\right\rangle^{2} d t^{2},
$$

and, due to the fact we are dealing with process in the stationary regime, the same result applies to the variance $\operatorname{Var}\left[d X(t+\tau)^{2}\right]$.

Therefore, from Eqs. (B5), (B6) and (B7), we have that correlation function given by Eq. (B1) is

$$
\operatorname{Corr}\left[d X(t)^{2} d X(t+\tau)^{2}\right]=\frac{\left\langle\sigma(t)^{2} \sigma(t+\tau)^{2}\right\rangle-\left\langle\sigma(t)^{2}\right\rangle^{2}}{3\left\langle\sigma(t)^{4}\right\rangle-\left\langle\sigma(t)^{2}\right\rangle^{2}}+\mathrm{O}(d t) .
$$

This expression contains two terms which must be also derived: $\left\langle\sigma(t)^{2} \sigma(t+\tau)^{2}\right\rangle$ and $\left\langle\sigma(t)^{4}\right\rangle$. Again, these terms can be obtained with the help of Novikov theorem. Lengthy and tedious calculations allow us write them in terms of other expressions presented above (cf. Eqs. (19) and (20)). The first one is

$$
\left\langle\sigma(t)^{2} \sigma(t+\tau)^{2}\right\rangle=2\left[\langle\sigma(t) \sigma(t+\tau)\rangle^{2}-\langle\sigma(t)\rangle^{4}\right]+\left\langle\sigma(t)^{2}\right\rangle^{2},
$$

while the second one is obtained from the first one by assuming $\tau=0$. That is:

$$
\left\langle\sigma(t)^{4}\right\rangle=3\left\langle\sigma(t)^{2}\right\rangle^{2}-2\langle\sigma(t)\rangle^{4},
$$

If we insert these averages to the correlation (B88), we prove the equality given by Eq. (B1) and finally obtain Eq. (42).

\section{APPENDIX C: AN ALTERNATIVE NOISE CORRELATION}

We recall that our model is given by a three-dimensional random process (8) - (10)

$$
\begin{aligned}
& d X(t)=\sigma(t) d W_{1}(t), \\
& d \sigma(t)=-\alpha[\sigma(t)-m(t)] d t+k d W_{2}(t), \\
& d m(t)=-\alpha_{0}\left[m(t)-m_{0}\right] d t+k_{0} d W_{3}(t),
\end{aligned}
$$

where $d W_{i}(t)=\xi_{i}(t) d t(i=1,2,3)$ are Wiener processes, i.e., $\xi_{i}(t)$ are zero-mean Gaussian white noises with crosscorrelation given by $\left\langle\xi_{i}(t) \xi_{j}\left(t^{\prime}\right)\right\rangle=\rho_{i j} \delta\left(t-t^{\prime}\right)$. Note that $\rho_{i j}=\rho_{j i}$ and $\rho_{i i}=1$. We have supposed that $W_{3}(t)$ is independent of $W_{1}(t)$ and $W_{2}(t)$. And we also have assumed that there exists a non zero correlation between $W_{1}(t)$ and $W_{2}(t)$ that was quantified by the parameter $\rho$.

This appendix modifies this framework by changing the existing correlations betweeen the Wiener processes $W_{i}$ $(i=1,2,3)$. We want to analyze the consequences on the model when we allow correlations between $W_{1}(t)$ and $W_{3}(t)$ instead of between $W_{1}(t)$ and $W_{2}(t)$. In this case, the correlation matrix is

$$
\left(\rho_{i j}\right)=\left(\begin{array}{ccc}
1 & 0 & \rho \\
0 & 1 & 0 \\
\rho & 0 & 1
\end{array}\right)
$$

where, again, $-1 \leq \rho \leq 1$.

One can easily see that this change in the correlation matrix does not affect most of the main statistical properties of the model although there is one important property that is modified: the leverage correlation.

According to the definition (22), the leverage correlation is quantified by

$$
\mathcal{L}(\tau) \equiv \frac{1}{Z}\left\langle d X(t+\tau)^{2} d X(t)\right\rangle
$$

where $X(t)$ is the zero-mean return and $Z=\left\langle d X(t)^{2}\right\rangle^{2}=m_{0}^{4}\left(1+\nu^{2}+\widehat{\nu}_{0}^{2}\right)^{2} d t^{2}$ (cf. Eq. 291). We need to calculate the correlation

$$
\left\langle d X(t+\tau)^{2} d X(t)\right\rangle=H(\tau)\left\langle\sigma(t) d W_{1}(t) \sigma(t+\tau)^{2} d W_{1}(t+\tau)^{2}\right\rangle .
$$

Its derivation is similar to the one given in Section III Since we follow the Itô convention, $d W_{1}(t+\tau)$ is independent of the rest of variables when $\tau>0$. This fact and the averages $\left\langle d W_{1}(t)\right\rangle=0$ and $\left\langle d W_{1}(t)^{2}\right\rangle=d t$ allow us to show that

$$
\left\langle d X(t+\tau)^{2} d X(t)\right\rangle=\left\langle\sigma(t) \sigma(t+\tau)^{2} d W_{1}(t)\right\rangle H(\tau) d t
$$




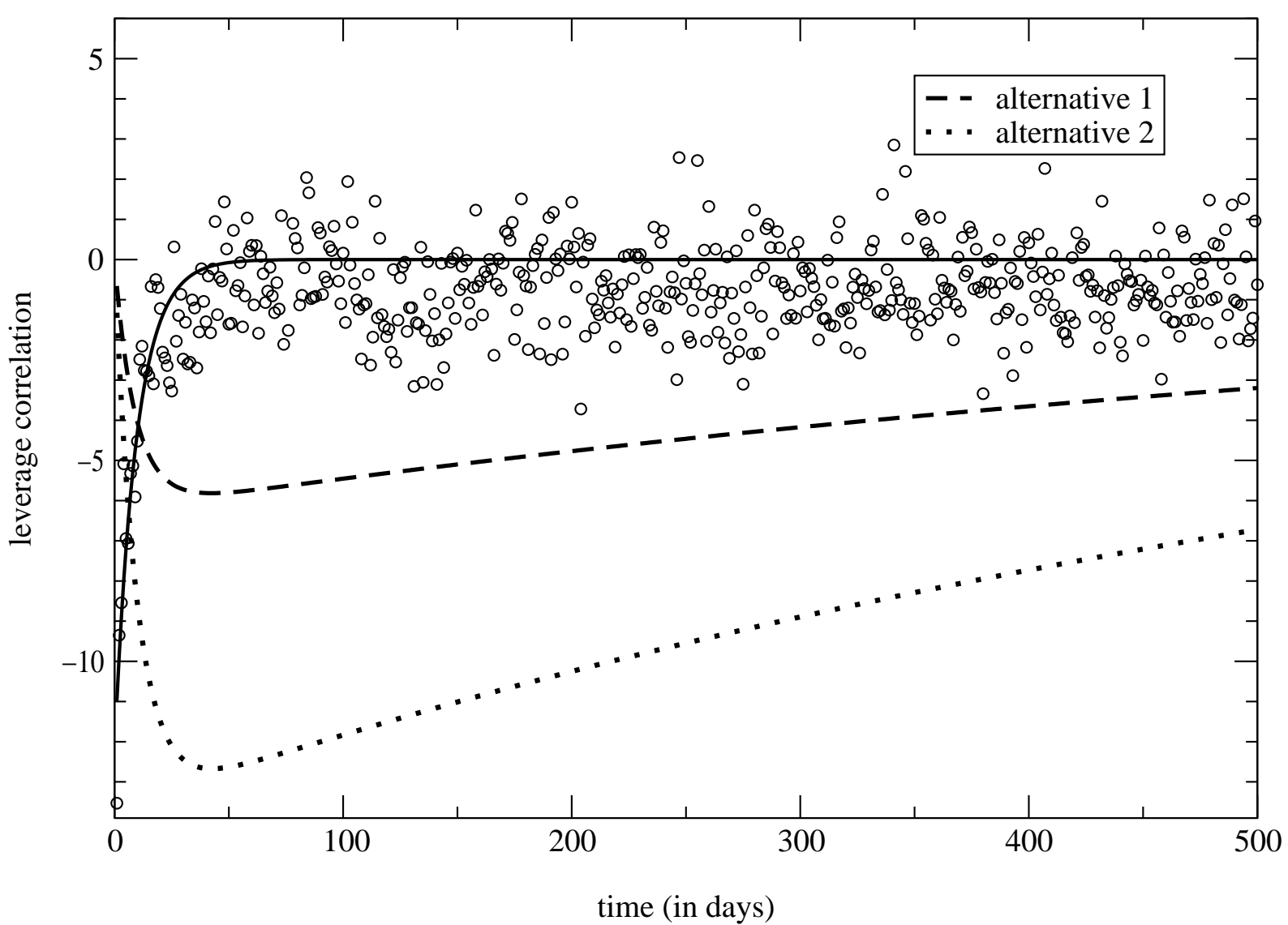

FIG. 5: The leverage effect in the Dow-Jones daily index with the alternative leverage. We plot the leverage function $\mathcal{L}(\tau)$ and $\overline{\mathcal{L}}(\tau)$ and compare with the Dow-Jones index from 1900 until 2000. The fit with the leverage function $\mathcal{L}(\tau)$ given by Eq. (30) takes parameters given by Table We also plot the two alternative leverage functions given by Eq. (C7). Alternative 1 shows the possible leverage with $\alpha=0.1, a=0.14, \alpha_{0}=0.04$ and $b=0.04$ while alternative 2 takes parameters $\alpha_{0}=0.1, b=0.14$, $\alpha=0.04$ and $a=0.04$.

where $H(\tau)$ is the Heaviside step function given by Eq. (27). The next step of the calculation needs to apply the Novikov theorem enunciated in Appendix $\mathbb{A}$ although now its result appears to be different from the one given by Eq. (28) since the correlation is between $W_{1}(t)$ and $W_{3}(t)$. In the present case the Novikov theorem given by Eq. (A3) allows us to write

$$
\left\langle\sigma(t) \sigma(t+\tau)^{2} d W_{1}(t)\right\rangle=\sum_{i=1}^{3} \rho_{1 i}\left\langle\frac{\delta\left[\sigma(t) \sigma(t+\tau)^{2}\right]}{\delta \xi_{i}\left(t^{\prime}\right)}\right\rangle d t .
$$

On the other hand we know from Eq. (14) that

$$
\sigma(t)=m_{0}+k \int_{-\infty}^{t} \xi_{2}\left(t^{\prime}\right) e^{-\alpha\left(t-t^{\prime}\right)} d t^{\prime}+\frac{k_{0}}{1-\lambda} \int_{-\infty}^{t} \xi_{3}\left(t^{\prime}\right)\left[e^{-\alpha_{0}\left(t-t^{\prime}\right)}-e^{-\alpha\left(t-t^{\prime}\right)}\right] d t^{\prime},
$$

where the integrals are represented in the Itô sense. Since volatility (C4) does not depend on $\xi_{1}$ and the only two non zero contributions correspond to $\rho_{11}=1$ and $\rho_{13}=\rho$ (c.f. Eq. (C1)),

$$
\left\langle\sigma(t) \sigma(t+\tau)^{2} d W_{1}(t)\right\rangle=2 \rho\left\langle\sigma(t) \frac{\delta \sigma(t+\tau)}{\delta \xi_{3}\left(t^{\prime}\right)}\right\rangle d t .
$$

But from Eq. C4

$$
\frac{\delta \sigma(t+\tau)}{\delta \xi_{3}(t)}=\frac{k_{0}}{1-\lambda}\left(e^{-\alpha_{0} \tau}-e^{-\alpha \tau}\right) \mathrm{H}(\tau)
$$


and $\langle\sigma(t)\rangle=m_{0}$. Hence the leverage correlation given by Eq. (C2) reads

$$
\overline{\mathcal{L}}(\tau)=\mathrm{H}(\tau) \bar{A}(\tau)\left(e^{-\alpha_{0} \tau}-e^{-\alpha \tau}\right),
$$

where

$$
\bar{A}(\tau) \equiv \frac{2 \rho \nu_{0} \sqrt{2 \alpha_{0}}}{m_{0}(1-\lambda)\left(1+\nu^{2}+\hat{\nu}_{0}^{2}\right)^{2}}\left[1+\left(\nu^{2}-\frac{\hat{\nu}_{0}^{2} \lambda}{1-\lambda}\right) e^{-\alpha \tau}+\frac{\hat{\nu}_{0}^{2}}{1-\lambda} e^{-\alpha_{0} \tau}\right] .
$$

We now compare the two leverage correlations $\mathcal{L}(\tau)$ and $\overline{\mathcal{L}}(\tau)$. In Section (31) and see that the limit $\mathcal{L}\left(0^{+}\right)$goes to a certain negative value. In contrast, in the present alternative model, $\overline{\mathcal{L}}\left(0^{+}\right)=0$. In Fig. [5] we see that leverage of the Dow-Jones index goes to -11.9 when $\tau \rightarrow 0^{+}$. Hence, this alternative model is in clear contradiction with empirical observations. In addition, we also observe in Fig. [5] that $\overline{\mathcal{L}}(\tau)$ has a longer range than the one observed in the Dow-Jones index. The reason for this being is that the exponential time decay of the leverage function (C8) is dominated by the same parameter as to the case of the volatility autocorrelation (43). Thus, in the present case, leverage and volatility correlations have the same long range persistence and this is also in clear contradiction with the empirical results telling us that leverage has a much shorter memory than the volatility

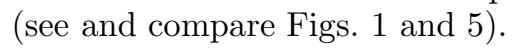

[1] M. Dacorogna, R. Gençay, U. Müller, R. Olsen, and O. Pictet, An Introduction to High-Frequency Finance (Academic Press, London, 2001).

[2] R. Cont, Empirical properties of asset returns: stylized facts and statistical issues, Quantitative Finance 1223 (2001).

[3] D. M. Guillaume, M. M. Dacorogna, R. D. Davé, U. A. Müller, R. B. Olsen and O. V. Pictet, From the bird's eye to the microscope: A survey of new stylized facts of the intra-daily foreign exchange markets Finance and Stochastics 195 (1997).

[4] R. Mantegna, H. E. Stanley, An Introduction to Econophysics (Cambridge University Press, Cambridge, 1999).

[5] A. Lo, Long term memory in stock market prices, Econometrica 591279 (1991).

[6] Z. Ding, C. W. J. Granger, and R. F. Engle, A long memory property of stock market returns and a new model, Journal of Empirical Finance, 183 (1993).

[7] Y. Liu, P. Cizeau, M. Meyer, C.-K. Peng, H. E. Stanley, Correlations in Economic Time Series, Physica A 245437 (1997).

[8] J. F. Muzy, J. Delour, and E. Bacry, Modelling fluctuations of financial times series: from cascade process to stochastic volatility model, The European Physical Journal B 17537 (2000); E. Bacry, J. Delour, J. F. Muzy, Multifractal random walk, Physical Review E 64026103 (2001).

[9] E. Stein and J. Stein, Stock Price Distributions with Stochastic Volatility: An Analytic Approach, Review of Financial Studies 4727 (1991); reprinted in Volatility: New Estimation Techniques for Pricing Derivatives, Robert Jarrow Ed., Risk Publications, 1998, pp. 325-339.

[10] S. Heston, A closed-form solution for options with stochastic volatiliy with applications to bond and currency options, Review of Financial Studies 6327 (1993).

[11] J.-P. Fouque, G. Papanicolaou, and K. R. Sircar, Derivatives in Financial Markets with Stochastic Volatility (Cambridge University Press, Cambridge, 2000).

[12] R. Schöbel and J. Zhu, Stochastic volatility with an Ornstein-Uhlenbeck process: An extension, European Finance Review 323 (1999).

[13] J. Masoliver and J. Perelló, A correlated stochastic volatility model measuring leverage and other stylized facts, International Journal of Theoretical and Applied Finance 5541 (2002).

[14] A. Dragulescu and V. Yakovenko, Probability distribution of returns in the Heston model with stochastic volatility, Quantitative Finance 2443 (2002).

[15] B. LeBaron, Stochastic volatility as a simple generator of apparent financial power laws and long memory, Quantitative Finance 1621 (2001).

[16] A. Fisher, L. Calvet, B. B. Mandelbrot, Multifractality of DEM/\$ rates, Cowles Foundation Discussion Paper 1165.

[17] L. Calvet, A. Fisher, Multifractality in asset returns: theory and evidence, Forthcoming in Review of Economics and Statistics.

[18] F. Black, Studies of stock market changes, Proceedings 1976 of the American Statistical Association, Business and Economics Statistics Section 177 (1976).

[19] G. Bekaert, G. Wu, Asymmetric volatility and Risk in Equity markets, The Review of Financial Studies 131 (2000).

[20] J.-P. Bouchaud, A. Matacz, and M. Potters, Leverage effect in financial markets: The retarded volatility model, Physical Review Letters 87228701 (2001).

[21] J. Perelló and J. Masoliver, Random diffusion and leverage effect in financial markets, forthcoming in Physical Review E (2003).

[22] B. Pochart and J.-P. Bouchaud, The skewed multifractal random walk with applications to option smiles, Quantitative Finance 2303 (2002).

[23] E. Bacry, J. F. Muzy, in preparation. 
[24] E. Bacry, J. Delour, J. F. Muzy, Modelling financial time series using multifractal random walks, Physica A 29984 (2001).

[25] S. Miccichè, G. Bonanno, F. Lillo, R. N. Mantegna, Volatility in financial markets: stochastic models and empirical results, e-print cond-mat/0202527

[26] J.-P. Bouchaud and M. Potters Theory of Financial Risks (Cambridge University Press, Cambridge, 2000), Theory of Financial Risk and Derivative Pricing (Cambridge University Press, Cambridge, 2003, forthcoming).

[27] R. Engle and A. Patton, What good is volatility model?, Quantitative Finance 1237 (2001).

[28] J. Perelló, J. M. Porrà, M. Montero, and J. Masoliver, Black-Scholes option pricing within It and Stratonovich conventions, Physica A 278260 (2000).

[29] T. Lux, The stable Paretian hypothesis and the frequency of large returns: an examination of major German stocks, Applied Financial Economics 6463 (1996).

[30] F. Longin, The Asymptotic Distribution of Extreme Stock Market Returns, Journal of Business, 69383 (1996)

[31] V. Plerou, P. Gopikrishnan, L. A. Amaral, M. Meyer, H.E. Stanley, Scaling of the distribution of price fluctuations of individual companies, Physical Review E 606519 (1999).

[32] J. Masoliver, M. Montero, J. M. Porrà, A dynamical model describing stock market price distributions, Physica A 283, 559-567 (2000).

[33] J. Masoliver, M. Montero, G. H. Weiss, Continuous-time random-walk model for financial distributions, forthcoming in Physical Review E (2003).

[34] E. A. Novikov, Functionals and the random-force method in turbulence theory, Soviet Physics JETP 20, 1290 (1965).

[35] P. Hanggi in Stochastic Processes Applied to Physics, L. Pesquera and M. A. Rodriguez Eds., 69-95, (World Scientific Press, Singapore, 1985). 